Z Rheumatol 2011 · 70:739

DOI 10.1007/s00393-010-0797-3

(c) Springer-Verlag 2011

\author{
O. Distler \\ Rheumaklinik, UniversitätsSpital Zürich
}

\title{
Endokrinologie und Rheumatologie
}

ge Rolle in der Pathogenese und Progredienz der Erkrankung. Die Autoren arbeiten in ihrem Artikel Unterschiede und Gemeinsamkeiten von Assoziationen und molekularen Schlüsselmechanismen heraus. Inwieweit Anti-Zytokin-Therapien bei Typ-1-Diabetikern ähnlich wie bei der rheumatoiden Arthritis sogar eine Therapieoption darstellen, wird in dem Beitrag ebenfalls dargestellt.

A. Maurer, A. Schwarting und G.J. Kahaly stellen in ihrem Beitrag die Polyglandulären Autoimmunsyndrome vor, bei denen es genetisch bedingt zu einer Störung des Immunsystems mit Schädigung endokriner Drüsen und anschließendem Funktionsverlust kommt. Für den Rheumatologen ist das Adulte Polyglanduläre Autoimmunsyndrom von besonderer Bedeutung, da hier Assoziationen mit der rheumatoiden Arthritis, dem Sjögren-Syndrom und dem systemischen Lupus erythematodes bestehen können. Die Autoren haben an der Medizinischen Universitätsklinik Mainz ein Register für Polyglanduläre Autoimmunsyndrome initiiert, was die Erkenntnis über Zusammenhänge mit rheumatologischen Autoimmunerkrankungen vertiefen wird.

Ein weiteres Gebiet, in dem Rheumatologen mit endokrinologischen Fragestellungen konfrontiert werden, ist die Myopathie-Abklärung. Die Kollegen S. Wenninger und B. Schoser arbeiten in ihrem Beitrag die anspruchsvolle Differentialdiagnose endokrinologischer Myopathien insbesondere auch im Hinblick auf Polymyositiden heraus. Besonders häufig und wichtig für den Rheumatologen ist hier die Steroidmyopathie, die in unterschiedlicher Ausprägung bei bis zu 20\% der Patienten auftreten kann, die längerfristig mit Prednison in einer Dosis $>10 \mathrm{mg} / \mathrm{d}$ behandelt werden .

Neue klinische und pathogenetische Aspekte des spannenden Gebietes der Neuroendokrinimmunologie bearbeitet R.H. Straub in seinem Beitrag. Der Artikel beschäftigt sich mit Folgekrankheiten chronisch entzündlicher Erkrankungen wie z.B. Fatigue, Anorexie/Mangelernährung, entzündungsassoziierte Anämie und Osteopenie. Er stellt eine neue, innovative Hypothese zur Entstehung dieser Folgekrankheiten unter Benutzung von Elementen der Evolutionsmedizin und der neuroendokrinen Regulation des Energiehaushalts vor. Die interessante Hypothese wird derzeit in weiteren experimentellen Modellen getestet.

Zusammenfassend zeigen die Artikel sowohl pathogenetische als auch klinische Gemeinsamkeiten und Überlappungen zwischen Endokrinologie und Rheumatologie. Manche von diesen Zusammenhängen sind neu und überraschend, andere sind bereits etabliert und für den klinischen Alltag von Bedeutung.

Ihr
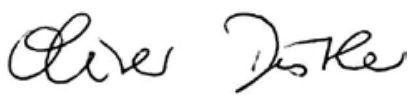

O. Distler

\section{Korrespondenzadresse PD Dr. O. Oliver Distler}

Rheumaklinik, UniversitätsSpital Zürich, Gloriastrasse 25, 8091 Zürich, Schweiz. oliver.distler@usz.ch matologischen Erkrankungen spielen proinflammatorischen Zytokine eine wichti- 